\title{
Experiencing Your Life: Increasing Self-Awareness through a Story-Inspired Paradigm
}

\author{
Dana Pavel, Matthias Holweg \\ Judge Business School \\ University of Cambridge \\ Cambridge, UK
}

\author{
Dirk Trossen \\ Computer Laboratory \\ University of Cambridge \\ Cambridge, UK
}

\begin{abstract}
Our demonstration showcases advances in lifestyle management, focusing on providing a platform able to record various information, process it and present it to end users through a novel story-inspired interface. We briefly outline the scope of this work and the envisioned benefits to the healthcare area before describing what we demonstrate and how we intend to engage with the conference participants.
\end{abstract}

\section{Keywords-Lifestyle management, storytelling}

\section{Why LifESTYLE MANAGEMENT?}

Driven by individual as well as societal changes and needs [1], lifestyle management has become an increasingly important area, both within research as well as within the growing space of innovative wellness-related solutions. As an important aspect of our work, we aim at complementing the current healthcare approaches with solutions that support end users' self-awareness and self-understanding with respect to their lifestyle and the impact their lifestyle choices have on their wellbeing. Since lifestyles are complex and evolving, it is not enough for systems aimed at this area to only focus on isolated aspects of users' lives. Instead, we need to take a more holistic and long-term view of what is important and try to capture as many aspects of people's lives as possible [2]. The aim of such approach is to better support users in self-awareness, self-understanding, self-reflection and, ultimately, self-change [3].

\section{WHAT IS DEMONSTRATED?}

In our work, we created a context-aware lifestyle management system, called MyRoR (a play on mirror). The main objective of MyRoR is to provide a more comprehensive picture of a user's daily life, supporting end users in identifying not only what happened during the day but also in providing objective clues related to why it might have happened. Two main aspects are important in achieving this objective: (1) we need to model, acquire, and corelate information from a variety of input sources, ranging from wearable sensors over mobile devices and laptop/ desktop devices to relevant Internet-based information; (2) we need to devise visualisation and interaction means that bring together the vast amount and variety of recordings within a compact and engaging format, allowing end users to examine, understand, own and share their experiences. In this, personalisation of both recording and visualisation means is important, as shown in [4]. The demonstration of MyRoR will specifically address these two aspects.
Let us briefly outline the challenge for the first aspect. MyRoR supports a variety of technological means to gather end user information. Figure 1 provides an overview of the plethora of information that is currently supported by the MyRoR system, ranging from wearable sensors to mobile devices (such as phone or tablet) to computing equipment such as desktop and laptops. The sources used as well as the input data recorded depend on the chosen scenario as well as on the end user's needs and constraints. An underlying information model supports any recording scenario. Given its ubiquity as well as increasing recording, processing and interaction capabilities, mobile phones play a major role in our platform. For collecting information from and through the phone we created and developed the AIRS platform [5], an Android-based application [6] able to provide access to more than 60 information sources on the mobile device or attached to it. New information sources can be easily added to the AIRS platform and therefore integrated into MyRoR overall. As Figure 1 outlines, we also utilise Internet-based sources, such as for the user's email and calendar information as well as for localised weather information.

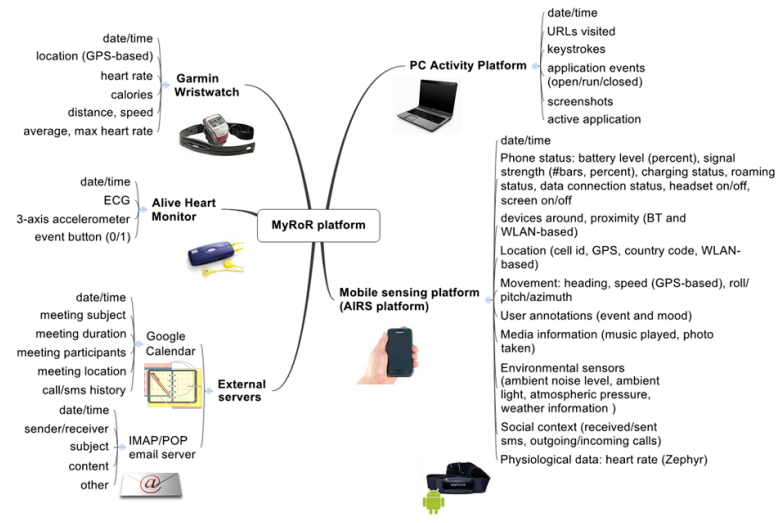

Figure 1. Information Sources in MyRoR

User-based annotations (e.g., via phone, Alive monitor or desktop) play an important role in the system and they are incorporated into the stories. Given the major privacy concerns involved in dealing with such data, the user information is stored and processed on a user-side machine, under the end user's full control. The variety of information we can gather and correlate allows for covering different inter-connected aspects of an end user's lifestyle. However, such variety also poses a major challenge on modeling and visualisation paradigms. For addressing this chalenge we 
found inspiration in familiar interfaces such as blogs as well as in creating stories as information structures of meaningful events. As we observed that end users can derive new and unexpected knowledge from reflecting over their data, it was also important to allow them to examine their data both at a detailed level (e.g., through graph or mapbased representations) as well as at an abstracted level (e.g., through stories or word clouds).

On the desktop side, the entry point into all of this data is provided through a Wordpress-based calendar interface where end users can select a day to look back on, experiencing the data available for this particular day in various forms. The MyRoR system supports detailed visualisations of individual data sources through timelines, tag clouds, detailed tables and context-enriched maps as well as our story-inspired visualisation method that combines many of the recorded information sources into a sequence of meaningful events, played back to the end user through a multimedia-enriched visualisation with media icons, text and personalised backgrounds (Figure 2 left).

On the mobile side, we have created a similar storyinspired interface (called Storica) that correlates information recorded through the AIRS platform. This interface also allows for a mixture of detailed and abstracted views into the processed user data (Figure 2 right).

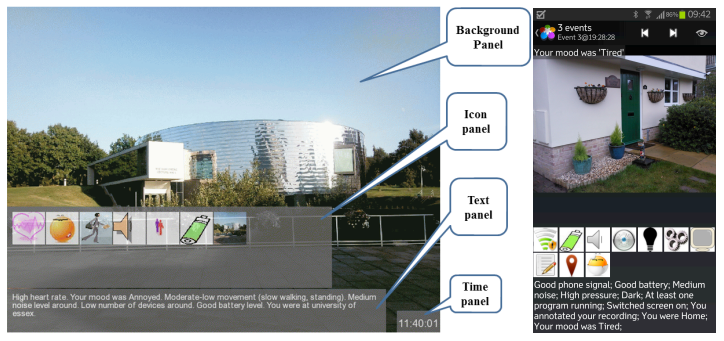

Figure 2. Story-based interface on desktop and mobile

Similar to the data capturing challenge, supporting the end user interaction over several devices is a challenge in itself. MyRoR provides several means for such multi-device support, which will be demonstrated. Firstly, the web-based desktop interface can be accessed through any (trusted) device, such as the user's tablet or mobile device, for visualisation purposes. This provides access to the entire pool of information, which is stored on the user's desktop (or laptop) machine. Secondly, we also demonstrate the visualisation on mobile devices directly through our Storica application that allows for accessing the information recorded through the AIRS platform. This mobile application employs adapted versions of the rich interactions provided by the desktop platform with the ability to visualise individual information through more detailed graphs, tag clouds and alike. Internet-based maps are used for visualising tracks with fly-over effects and the ability to enquire about context information at any recorded location.

Figure 3 provides an overview of our demonstrations of the MyRoR system, spanning from desktop to mobile devices as well as from the recording phase to the visualisation across devices.

\section{How Do We INTERACT WITH THE PARTICIPANTS?}

Given the public availability of the AIRS platform [6], we encourage participants to install, record and annotate their conference experiences on their mobile device.

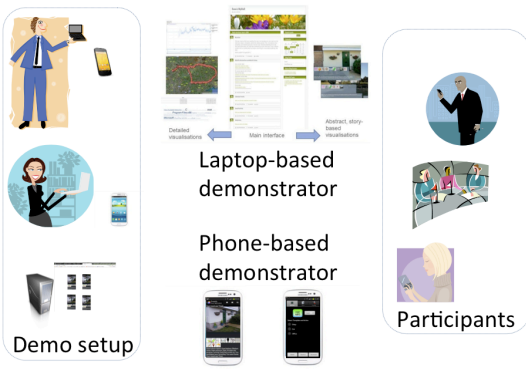

Figure 3. Scope of demonstration

In preparation for the demonstration, we have made the Storica application [7] available to participants, enabling people to visualize individual experiences on their mobile. We encourage the sharing of these experiences through various channels, such as Twitter, Google+ and Facebook or the dedicated sharing space set up for the conference. In collaboration with the organizers, we have disseminated instructions to the participants via a leaflet. With that, participants not only directly experience the platform but also create and possibly share experiences of the conference. We also plan to link these experience channels to the conference website for further dissemination. In addition, we demonstrate the desktop-based platform during the demonstration session with visualizations of the authors' data over a prolonged period of time (recording in various forms has been ongoing for almost two years). These desktop-based experiences will complement the shared experiences by participants throughout the conference event.

\section{ACKNOWLEDGMENT}

The MyRoR work has been funded by UK's TSB and EPSRC under project no. TP/AN072C and by a seed fund study at Judge Business School.

\section{REFERENCES}

[1] Future Foundation and Friends Provident, "Visions of Britain 2020: Health and Wellbeing," 2010.

[2] D. T. Wade, "Holistic Health Care: What is it, and how we can achieve it?," 2009.

[3] B. J. Fogg, Persuasive Technologies: Using computers to change what we think and do. San Francisco, CA: Morgan Kaufmann, 2003.

[4] D. Pavel, V. Callaghan, A. K. Dey, F. Sepulveda, M. Gardner, "The Story of Our Lives: From Sensors to Stories in Self-monitoring Systems," in The 4th Computer Science and Electronic Engineering Conference (CEEC'12), Colchester, UK, 2012.

[5] D. Trossen, D. Pavel, “AIRS: A Mobile Sensing Platform for Lifestyle Management Research and Applications," 5th Conference on MOBILe Wireless MiddleWARE, Operating Systems, and Applications (MobilWare 2012), November 2012, Berlin, Germany.

[6] AIRS, at https://play.google.com/store/apps/details?id=com.airs, 2013.

[7] Storica, at https://play.google.com/store/apps/details?id=com.storica, 2013 